Methods and results We evaluated 3691 patients treated with drugeluting stents who had a baseline CRP measurement. The primary outcome was stent thrombosis; secondary outcomes were death, myocardial infarction (MI), death or MI, and target vessel revascularization. During follow-up (median, 2 years), 26 patients had definite or probable stent thrombosis, 146 patients died, 239 had an MI, and 206 underwent target vessel revascularization. In multivariable Cox proportional-hazards models, elevated levels of hs-CRP were significantly associated with increased risk of stent thrombosis. Elevated hs-CRP levels also significantly predicted the risks of death, MI, and death or MI, but not target vessel revascularization.

Conclusions Elevated hs-CRP levels were significantly associated with increased risks of stent thrombosis, death, and MI in patients receiving drug-eluting stents, suggesting the usefulness of inflammatory risk assessment with CRP.

\section{e0407 RESULTS OF SKELETONISED BIMA IN COMBINATION WITH} GEA IN CABG

doi:10.1136/hrt.2010.208967.407

Biao Yuan, Mingkui Zhang, Yongqiang Jin, Shen Zhao, Qingyu Wu. First Hospital of Tsinghua University

Objective To summarise and analyse the clinical experience in the use of skeletonised bilateral internal mammary artery in combination with right gastroepiploic artery in coronary artery bypass grafting.

Methods A retrospective review was made to 112 patients underwent Off- Pump Coronary Artery Bypass Grafting since January 2007 to may 2009. The patients were 79 men and 33 women, with a mean age of 67 years (range 42-81 years).

Result Used 112 skeletonised LIMA, with or without sequential grafting, in-situ BIMA, GEA and RA were used in 81(72\%), 104 $(93 \%)$ and $59(53 \%)$ patients respectively. The mean number of distal anastomoses per patient was 3.43(range 2-5), Compssite $\mathrm{Y}$ or $\mathrm{T}$ graft was constructed in 41 patients, Sequential anatomoses was performed in 34 patients, Aortic no touch technique was used in 53 patients. There was no hospital mortality, no the perioperative myocardial infarction and sternal wound complication.

Conclusion Skeletonized BIMA in combination with the GEA is a very versatile situ conduit to achieve complete arterial revascularization. The only constraindications for the use of BIMA and GEA grafts were emergency operations with haemodynamic instability.

\section{e0408 A CLINICAL ANALYSIS OF ACUTE MYOCARDIAL INFARCTION IN YOUNG PATIENTS}

doi:10.1136/hrt.2010.208967.408

Fu Xianghua, Wu Weili, Zhang Wenjing, Wang Xuechao, Wang Yanbo, Jiang Yunfa, Hao Guozhen. The 2nd Hospital of Hebei Medical University, Shijiazhuang, Hebei, China

Objective To investigate the clinical characteristics of acute myocardial infarction (AMI) in young patients.

Methods We carried out the contrasting analysis in the clinical data between 45 young patients (age $\leq 45$ years old) and 52 old patients (age $\geq 60$ years old).

Results Young AMI patients were often male, and had the typical clinical manifestations. The smoking rate hyperfibrinogenemia rate and positive family history rate of the young people group were markedly higher than those of the old people group $(p<0.05)$. The morbidity rate of patients with single coronary artery atherosclerosis was high in the young people group. The morbidity rate of patients with multiple coronary artery atherosclerosis was high in the old people group. The patients in the old people group who complicated with cardiac aneurysm, arrhythmia, heart failure, cardiac shock were much more than those in the young people group $(\mathrm{p}<0.05)$.

Conclusion Smoking, hyperfibrinogenemia and positive family history are main causes of AMI in young patients. Young AMI patients had the typical clinical manifestations with simple coronary lesion. The complications in the young people group are less than those in the old people group, and the prognosis was better than old cases.

\section{e0409 INFLUENCE ON THE PLATELET FUNCTION OF DIFFERENT STATINS COMBINED WITH LOADING DOSE CLOPIDOGREL IN PATIENTS WITH ACUTE CORONARY SYNDROME}

doi:10.1136/hrt.2010.208967.409

Fu Xianghua, Wang Qian, Wang Yanbo, Wang Xuechao, Hao Guozhen, Jiang Yunfa, Gu Fan WeizeXinshun. The 2nd Hospital of Hebei Medical University, Shijiazhuang China

Objective To investigate clinical effects of clopidogrel combined with simvastatin or fluvastatin on the platelet aggregation rate (PAR), platelet activation marker CD62P and the incidence of major adverse cardiovascular events (MACE) in patients with ACS.

Methods From April 2008 to December 2009, one hundred patients (79 male and 21 female, average age $61.46 \pm 12.84$ years) who had been diagnosed as ACS were enrolled into this study. These cases were randomly divided into two groups, the Group $A(n=50$, treated with simvastatin $20 \mathrm{mg}$ per night); the Group B ( $n=50$, treated with fluvastatin $40 \mathrm{mg}$ per night). Detailed clinical information was collected. PAR, CD62P, alanine aminotransferase (ALT), and aspartate aminotransferase (AST) of the two groups were measured. All cases received clopidogrel (a loading dose of $300 \mathrm{mg}$ and then $75 \mathrm{mg}$ daily), aspirin and Low molecular weight heparin. The MACE within 14 days were recorded.

Result there was no significant differences in baseline between the Group A and Group B. There was no significant differences in the PAR and expression rate of CD62P after $300 \mathrm{mg}$ clopidogrel ( $p>0.05) .1 \mathrm{~h}$ after treated with statins the expression rate of CD62P and PAR in the two groups were lower than that before treated with statins $(p<0.05)$. After $14 d$ treated with statins the expression rate of CD62P and PAR were still lower than that before treated with statins $(p<0.05)$. There were no significant increase of ALT and AST in the both groups $(p>0.05)$. After the above-mentioned medical treatment, the expression rate of $\mathrm{CD} 62 \mathrm{P}$ and PAR in the two groups were similar $(p>0.05)$. There were no significant differences in the incidence of MACE between two groups.

Conclusion ACS patients with loading dose clopidogrel combined with simvastatin or fluvastatin could decrease the MACE, the results in two groups are similar. Neither simvastatin with clopidogrel nor fluvastatin with clopidogrel decreases the platelet activity of clopidogrel.

\section{e0410 ADVERSE EFFECTS OF GLIBENCLAMIDE ON MYOCARDIA PERFUSION IN PATIENTS WITH ACUTE MYOCARDIAL INFARCTION AND TYPE 2 DIABETES MELLITUS}

doi:10.1136/hrt.2010.208967.410

F u Xianghua, W u Weili, Yan Yangmei, Wang Xuechao, Wang Yanbo, Fan Weize Jiang Yunfa, Hao Guozhen. The 2nd Hospital of Hebei Medical University, Shijiazhuang, Hebei, China

Objective To assess the adverse effects of glibenclamide on the myocardium, for investigating more effective and rational therapy. 